\title{
Article
}

\section{A BIST Scheme for Bootstrapped Switches}

\author{
Xiao-Bin Tang * and Masayoshi Tachibana
}

check for

updates

Citation: Tang, X.-B.; Tachibana, M. A BIST Scheme for Bootstrapped Switches. Electronics 2021, 10, 1661. https://doi.org/10.3390/

electronics10141661

Academic Editor: Costas Psychalinos

Received: 4 June 2021

Accepted: 11 July 2021

Published: 12 July 2021

Publisher's Note: MDPI stays neutral with regard to jurisdictional claims in published maps and institutional affiliations.

Copyright: (C) 2021 by the authors. Licensee MDPI, Basel, Switzerland. This article is an open access article distributed under the terms and conditions of the Creative Commons Attribution (CC BY) license (https:/ / creativecommons.org/licenses/by/ $4.0 /)$.
Electronic and Photonic Systems Engineering, Kochi University of Technology, Kami, Kochi 782-8502, Japan; tachibana.masayoshi@kochi-tech.ac.jp

* Correspondence: 248003c@gs.kochi-tech.ac.jp; Tel.: +81-80-2066-9597

\begin{abstract}
This paper proposes a built-in self-test (BIST) scheme for detecting catastrophic faults in bootstrapped switches. The clock signal and the gate voltage of the sampling MOS transistor are taken as the observation signals in the proposed BIST scheme. Usually, the gate voltage of the sampling MOS transistor is greater than or equal to the supply voltage when the switch is turn on, and such a voltage is not suitable for observation. To solve this problem, a low power supply voltage is provided for the bootstrapped switch to obtain a suitable observation voltage. The proposed BIST scheme and the circuit under test (CUT) are realized with transistor level. The proposed BIST scheme was simulated by HSPICE. The simulated fault coverage is approximately $87.9 \%$ with 66 test circuits.
\end{abstract}

Keywords: built-in self-test; fault diagnosis; bootstrapped switches

\section{Introduction}

Fault diagnosis is an important element in the design and test of integrated circuits. Especially with the development of CMOS circuits, it is more and more difficult to detect faults only with limited input and output ports. BIST is a viable approach that has been used by many researchers [1-9].

For data converter fault diagnosis schemes, there are schemes based on Differential Non-Linearity (DNL) test data [1], based on code-width [2], based on a resistance matching [3], and so on. For amplifier fault diagnosis schemes, there is a scheme by using an RF peak detector and two comparators [4], a scheme by checking the stable output of transient response [5], a scheme based on chaotic oscillation [6], and so on. For sample and hold circuit fault diagnosis schemes, there is a scheme by online balance self-checking [7], a scheme by measuring performance parameters [8], a scheme by monitoring the same output with common-mode input [9], and so on.

Bootstrapped switches are widely used in many mixed-signal circuits [10-13]. For example, they are used in sample and hold circuits to achieve rail-to-rail switching functions $[10,11]$, used in charge pump circuits to improve energy harvesting by node precharging [12,13], and so on. There are also reports on improving the performance of bootstrap switches [14-16]. For example, the body effect compensation technology is used to improve the linearity of the bootstrapped switch $[14,15]$, the dual-channel sampling switch technology is used to improve the accuracy and linearity of the bootstrapped switch [16], and so on. However, there are few reports on the fault diagnosis schemes for bootstrapped switches.

Consequently, this paper proposes a BIST scheme for detecting catastrophic faults in bootstrapped switches. Section 2 previews the bootstrapped switch under testing. Section 3 presents the model of fault diagnosis. Section 4 presents the BIST implementation. Section 5 sets the simulation result by HSPICE. Section 6 discusses the results. Section 7 sets the conclusion.

\section{The Bootstrapped Switch under Test}

In this study, the bootstrapped switch in [17] was used as the circuit under test. The circuit and the designed size of elements are shown in Figure 1. CLK is the clock 
signal, which controls the "ON" phase and "OFF" phase of the bootstrapped switch. Vin is the input signal. Vout is the output signal. $V_{G}$ is the gate voltage of the sampling MOS transistor.

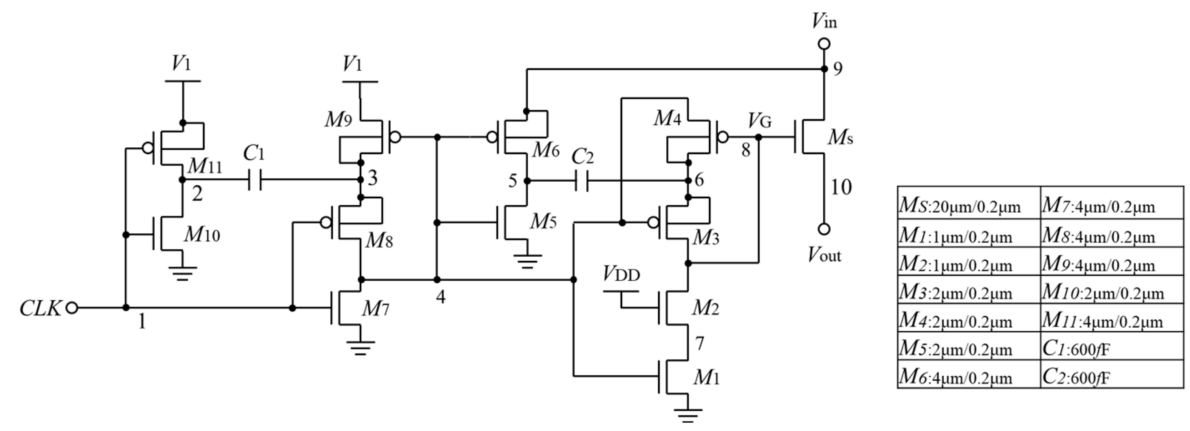

Figure 1. The bootstrapped switch in [17] and the designed size of elements.

When the bootstrapped switch is in the "ON" phase, the CLK is " 1 ". Then, the voltage of Node 4 is pulled down to GND via $M_{7}$ and the voltage of Node 2 is pulled down to GND via $M_{10}$. Then, the voltage of Node 3 is pulled up to $V_{D D}$ via $M_{9}$, the voltage of Node 5 follows the voltage of $V_{\text {in }}$ via $M_{6}$ and the voltage of Node 8 follows the voltage of Node 6 via $M_{3}$. The voltage of Node 5 is transmitted to Node 6 via $C_{2}$, so the voltage of Node 6 is the voltage of $V_{\text {in }}$ plus the voltage previously-stored at node 6 .

When the bootstrapped switch is in the "OFF" phase, the CLK is " 0 ". Then, the voltage of Node 2 is pulled up to $V_{D D}$ via M11 and the voltage of Node 4 follows the voltage of Node 3 via M8. Then, the voltage of Node 3 is pulled up to $2 V_{D D}$ via $C_{1}$, so the voltage of Node 4 is $2 V_{D D}$. Then, the voltage of Node 8 is pulled down to GND via $M_{2}$ and $M_{1}$. Then, the voltage of Node 6 follows the voltage of Node 4 via $M_{4}$.

So, the voltage of Node 6 is $2 V_{D D}$ in the "OFF" phase and $2 V_{D D}$ plus the voltage of $V_{\text {in }}$ in the "ON" phase.

The Bootstrapped technique improves linearity by increasing the voltage at the node to lower and stabilize the on-resistance of the sampling switch. In this study, the transmission voltage reached $2 V_{D D}$, which was difficult to set up the test circuit.

\section{The Proposed BIST Scheme}

When the bootstrapped switch is in the "ON" phase, the gate voltage of the sampling MOS transistor has a stable voltage. When the bootstrapped switch in the "OFF" phase, the gate voltage of the sampling MOS transistor is GND. According to this characteristic, the gate voltage of sampling MOS transistor and CLK signal are used as observation signals.

Due to the bootstrapped switch in this study, in the "ON" phase, the gate voltage of the sampling MOS transistor is $2 V_{D D}$. If the power supply voltage is $1.8 \mathrm{~V}$, then the gate voltage of the sample MOS transistor is $3.6 \mathrm{~V}$. Such a high voltage is not suitable for observation.

To solve this problem, a low power supply voltage is provided to the bootstrapped switch. As a result, an appropriate voltage can be obtained on the sampling MOS transistor during the "ON" phase.

The testing strategy is if the response analysis module meets the following conditions, output " $\mathrm{F}=0$ ", it indicates that the circuit is fault-free; otherwise output " $\mathrm{F}=1$ ", indicates that the circuit has faults.

1. When "CLK $=1$ ", the gate voltage of the sampling MOS is a constant value. In this study, the value is determined by a window comparator.

2. When "CLK $=0$ ", the gate voltage of the sample MOS is GND. In this study, the GND is determined by a phase inverter.

The proposed BIST schematic is shown in Figure 2. The CLK signal of the bootstrapped switch and the gate voltage of the sampling MOS $\left(V_{G}\right)$ are connected to the response analysis module as the input. The response analysis module analyzes the two-observation 
signal to determine whether the circuit has a fault. The $E N$ is an enabling signal that controls whether the analysis module works. The power supply voltage of the bootstrapped switch is $V D D_{1}$. The power supply voltage of the response analysis is $V_{D D 2}$.

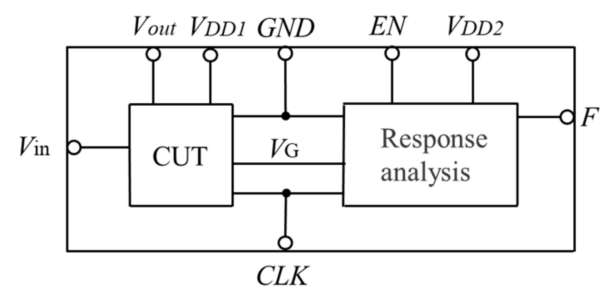

Figure 2. The proposed BIST schematic.

The schematic of the response analysis module is shown in Figure 3. When " $E N=0$ ", the response analysis module is not working. The $C L K$ signal and $V_{G}$ signal do not affect the response analysis module. The output $F$ is " 1 ". When " $E N=1$ ", the analysis module works. If the output is " 1 ", it indicates that the circuit is fault-free. If the output is " 0 ", it indicates that the circuit has faults. Because the bootstrapped switch is sensitive to the changes of $V_{G}$ signal, a source follower is used to collect the $V_{G}$ signal.

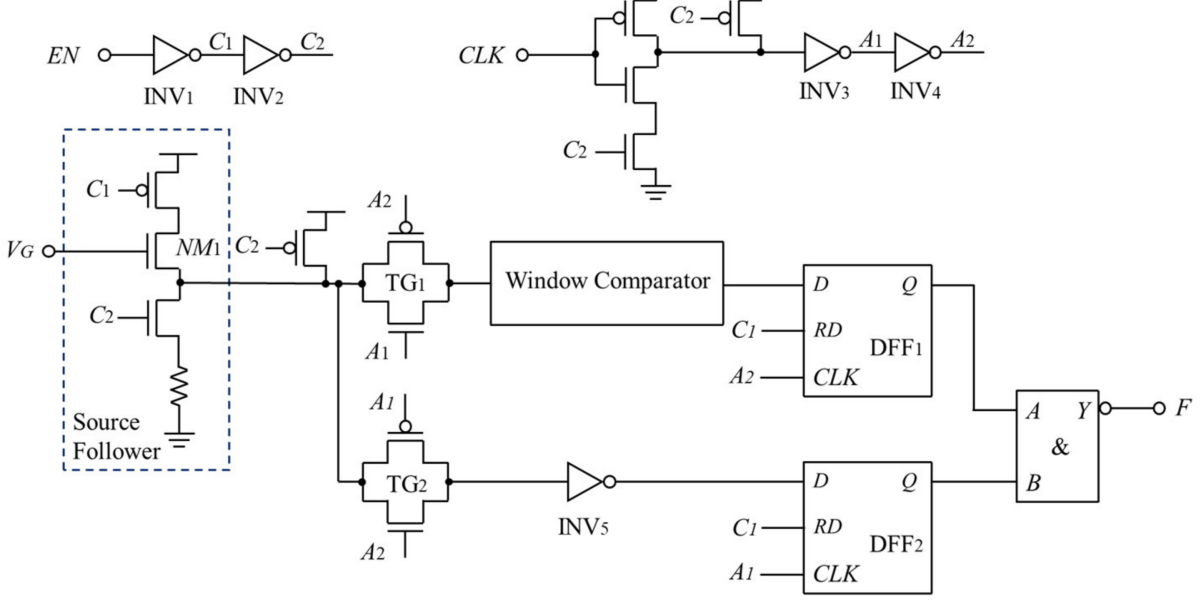

Figure 3. The schematic of the response analysis module.

The schematic of the window comparator in [5] is shown in Figure 4. The window comparator outputs a high potential within the designed input range. The input range can be adjusted by adjusting the $\mathrm{W} / \mathrm{L}$ of the four inverters.

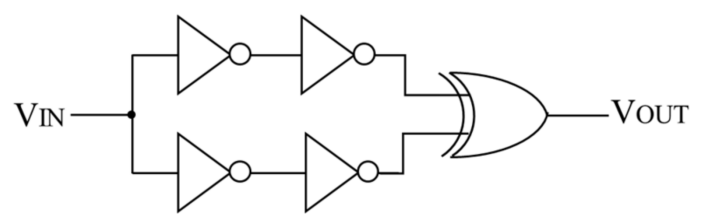

Figure 4. The schematic of the window comparator.

\section{Fault Modeling}

To evaluate the proposed test strategy, six fault models were added to the circuit under testing. The fault models considered in this paper are shown in Figure 5. 


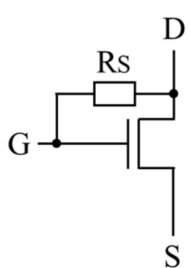

(a)

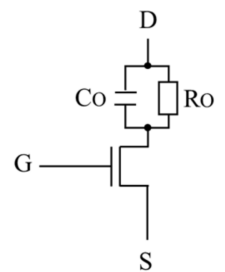

(d)

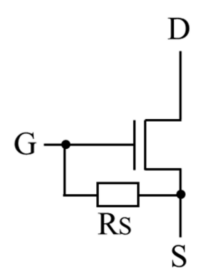

(b)

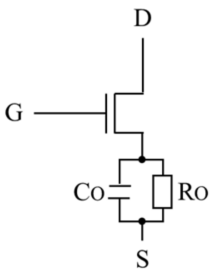

(e)<smiles>CCCC1C(C)CC(S)CC1C</smiles>

(c)

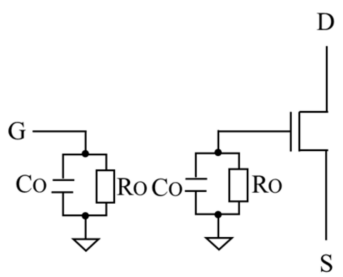

(f)

Figure 5. The six fault models in this paper (a) gate-drain short (GDS); (b) gate-source short (GSS); (c) drain-source short (DSS); (d) drain open (DO); (e) source open (SO); (f) gate open (GO).

The short fault of MOS transistor is simulated by a $10 \Omega$ resistor between two ports connected. The specific ones are Figure $5 \mathrm{a}$ gate-drain short (GDS), Figure $5 \mathrm{~b}$ gate-source short (GSS), and Figure 5c drain-source short (DSS), respectively. The open fault of MOS transistor is simulated by inserting a $100 \mathrm{fF}$ capacitor and a $10 \mathrm{M} \Omega$ resistor in parallel at the port. The specific ones are Figure $5 \mathrm{~d}$ drain open (DO), Figure 5 e source open (SO), and Figure $5 \mathrm{f}$ gate open (GO), respectively.

\section{Simulation Results}

To obtain the influence information of BIST on the bootstrapped switch, the performance simulation of the bootstrapped switch with and without the test circuit was carried out. As shown in Figure 6, the bootstrapped switch and a $20 \mathrm{pF}$ capacitor are combined into a track and hold circuit. The CUT schematic is shown in Figure 1. The schematic of CUT with BIST is shown in Figure 2.

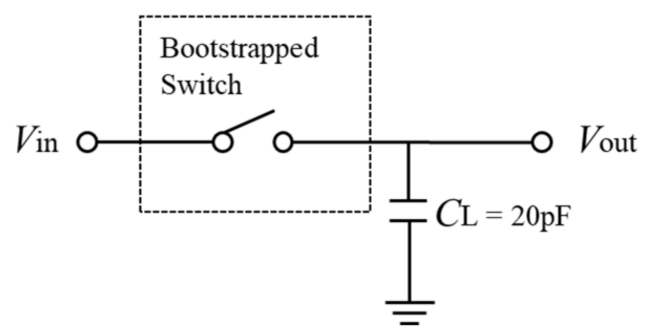

Figure 6. The performance simulation schematic for bootstrapped switch.

The CLK signal is a $10 \mathrm{M} \mathrm{Hz}$ square wave and the input signal is a sine wave with an amplitude of $1.8 \mathrm{~V}$ and a frequency of $4.96 \mathrm{M} \mathrm{Hz}$. After the spectrum analysis of the circuit, the performance parameters in Table 1 were obtained. The simulation results show that the BIST scheme has no obvious burden on the CUT. Note a slight decrease in SNR, which is due to the additional noise provided by $N M_{1}$. The $V_{G S}$ of the $M_{S}$ with BIST scheme is higher than that of the $M_{S}$ without the BIST scheme, which results in the on-resistance of the $M_{S}$ with the BIST scheme being lower than that of the $M_{S}$ without the BIST scheme. Therefore, the parameters except SNR are slightly improved. 
Table 1. Parameters comparison with and without BIST circuit.

\begin{tabular}{ccc}
\hline Parameter & CUT & CUT with BIST \\
\hline ENOB (bits) & 9.45 & 9.46 \\
SINAD $(\mathrm{dB})$ & 58.69 & 58.73 \\
SNR $(\mathrm{dB})$ & 83.35 & 83.23 \\
SFDR $(\mathrm{dBc})$ & 58.94 & 58.98 \\
THD $(\mathrm{dB})$ & -58.70 & -58.74 \\
\hline
\end{tabular}

To evaluate the proposed BIST scheme, a fault attachment script was used to add the six faults to the MOS transistor of the bootstrapped switch except for the $M_{S}$. The fault of DSS, DO and SO of $M_{S}$ does not cause voltage changes at the gate voltage of $M_{S}$ which as observation voltage. Therefore, this BIST scheme cannot detect the fault of DSS, DO and SO of $M_{S}$. However, the $M_{S}$ connects the input and output signals, a fault in the $M_{S}$ can be easily detected by other methods. Therefore, the $M_{S}$ is not used as a test object. The total number of fault circuits is 66 .

The $V_{D D 1}$ and the input signal are set to $0.6 \mathrm{~V}$, thus the $V_{G}$ is approximately $1.56 \mathrm{~V}$ during the "ON" phase. Therefore, the output voltage of the source follower is carefully designed to be approximately $0.7 \mathrm{~V}$, when the input voltage of the source follower is approximately $1.56 \mathrm{~V}$. To cope with the possible deviation of the circuit manufacturing, the window voltage of the window comparator is designed to be $0.5 \mathrm{~V}$ to $0.9 \mathrm{~V}$.

Figure 7 shows the output signal by transient simulation. Figure 7 a shows the result of fault-free. When BIST is off, the output is " $F=1$ "; when BIST is on, the output is " $F=0$ " which indicates that the circuit is fault-free. Figure $7 \mathrm{~b}$ shows the result of a fault with gate-source short with the $M_{1}$. When BIST is off, the output is " $F=1$ "; when BIST is on, the output is " $F=1$ " which indicates that the circuit has faults.

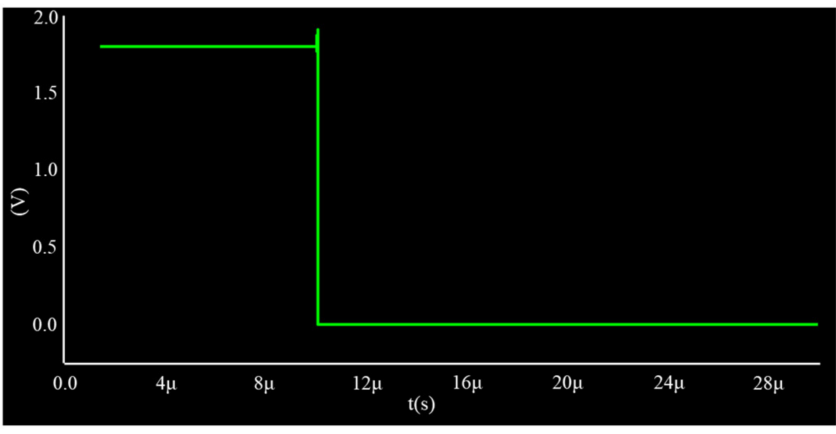

(a)

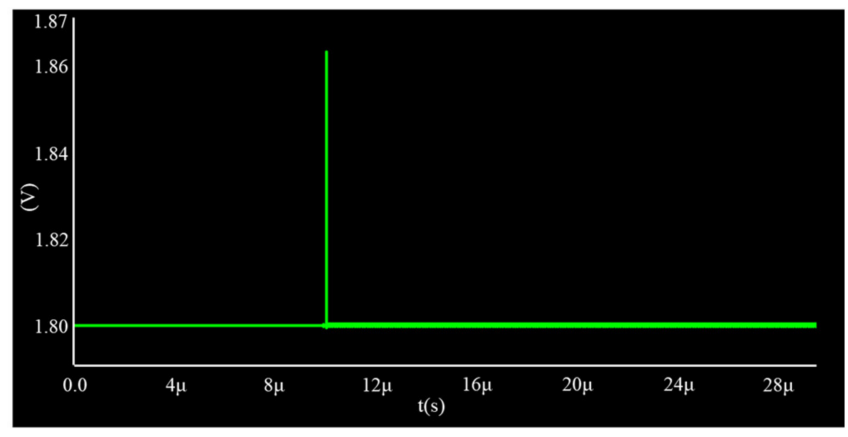

(b)

Figure 7. The output signal by transient simulation (a) fault-free; (b) fault with gate-source short with the $M_{1}$.

Table 2 shows the simulation results with 66 test circuits. The fault coverage of the BIST scheme is approximately $87.9 \%$. The 200 times Monte-Carlo simulation results show that the BIST scheme works well. Extreme process simulation results show that the fault coverage of the BIST scheme is approximately $75.8 \%, 78.8 \%, 89.4 \%$ and $84.8 \%$ at FF, FS, SS and SF corner, respectively. 
Table 2. Simulation results of injected and defected fault.

\begin{tabular}{ccc}
\hline Fault Types & Injected Faults & Detected Faults \\
\hline GDS & 11 & 11 \\
GSS & 11 & 11 \\
DSS $^{1}$ & 11 & 10 \\
GO $^{2}$ & 11 & 4 \\
DO & 11 & 11 \\
SO & 11 & 11 \\
\hline
\end{tabular}

${ }^{1}$ The undetected MOS transistors is $M_{2} .{ }^{2}$ The undetected MOS transistors are $M_{1}, M_{2}, M_{3}, M_{4}, M_{5}, M_{9}$ and $M_{10}$.

\section{Discuss}

The function of $M_{2}$ is to relieve the pressure of $M_{1}$, that is, to prevent the drain-source voltage of M1 from being greater than $V_{D D} . M_{2}$ can be equivalent to resistance. Therefore, the BIST scheme cannot be detected to the fault of DSS of $M_{2}$.

Note that the BIST scheme has low fault coverage for the gate open of the MOS transistor. This issue is discussed with the fault of GO of $M_{1}$.

Figure 8 shows the transient simulation of the gate voltage of $M_{1}$ with the fault of GO with $M_{1}$. Although the gate of $M_{1}$ is open, the gate voltage of $M_{1}$ is still high and low at the right time sequence due to the presence of parasitic capacitance. Therefore, $M_{1}$ can be turned on and off at the correct timing with the gate open. This indicates that the effect of this type of fault on the circuit is not catastrophic. However, this fault may still affect the circuit performance, and subsequent research will be carried out to address this issue.

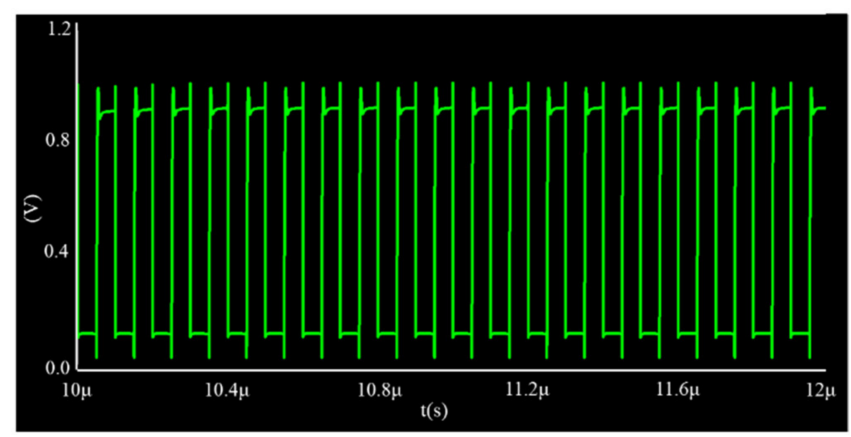

Figure 8. The gate voltage of M1 with the fault of GO of M1.

\section{Conclusions}

Bootstrapped switches are an indispensable type in analog circuits, but there are few fault diagnosis schemes for them. Thus, this paper proposed a bootstrapped switch BIST scheme for detecting catastrophic faults. According to the characteristics of the bootstrap switch, the clock signal and the gate voltage of the sampling MOS transistor are used as the observation signals. However, the gate voltage of the sampling MOS transistor reaches $2 V_{D D}$ during sampling, which is not conducive to observation. In this paper, the method of low power supply voltage is adopted to solve this problem. The proposed BIST scheme was designed and simulated in $0.18 \mu \mathrm{m}$ CMOS technology. The HSPICE simulation results show that the fault coverage is approximately $87.9 \%$ with 66 test circuits. However, in the real circuit, the test result of this BIST scheme may be lower than the simulation results due to noise, technology, or any uncertainty. Therefore, we plan to test and evaluate the prototype chip after it is delivered in the future.

Author Contributions: Conceptualization, X.-B.T. and M.T.; methodology, X.-B.T.; validation, M.T.; resources, M.T.; writing—original draft preparation, X.-B.T.; writing—review and editing, X.-B.T. and M.T.; visualization, X.-B.T.; supervision, M.T.; project administration, M.T.; funding acquisition, M.T. Both authors have read and agreed to the published version of the manuscript.

Funding: This research was funded by JSPS KAKENHI, grant number JP21K11813. 
Acknowledgments: This work was supported by VLSI Design and Education Center (VDEC), the University of Tokyo in collaboration with Synopsys, Cadence Design System, Mentor Graphics, Rohm Corporation and Toppan Printing Corporation.

Conflicts of Interest: The authors declare no conflict of interest.

\section{References}

1. Charoenrook, A.; Soma, M. Fault diagnosis of flash ADC using DNL test. In Proceedings of the IEEE International Test Conference-(ITC), Baltimore, MD, USA, 17-21 October 1993; pp. 680-689.

2. Lee, D.; Yoo, K.; Kim, K.; Han, G.; Kang, S. Code-width testing-based compact ADC BIST circuit. IEEE Trans. Circuits Syst. II Express Briefs 2004, 51, 603-606. [CrossRef]

3. Yuan, J.; Tachibana, M. A resistance matching based self-testable current-mode R-2R digital-to-analog converter. IEICE Electron. Express 2013, 10, 1-7. [CrossRef]

4. Ayadi, R.; Masmoudi, M. A new built-in self-test (BIST) for a RF low-noise amplifier (LNA). In Proceedings of the 5th International Conference on Design \& Technology of Integrated Systems in Nanoscale Era, Al Hammamat, Tunisia, 23-25 March 2010; pp. 1-5.

5. Yuan, J.; Tachibana, M. A BIST scheme for operational amplifier by checking the stable output of transient response. In Proceedings of the 20th European Conference on Circuit Theory and Design (ECCTD), Linkoping, Sweden, 29-31 August 2011; pp. 885-888.

6. Wannaboon, C.; Nattagit, J.; Tachibana, M. Chaotic oscillation-based BIST for CMOS operational amplifier. In Proceedings of the 2014 International SoC Design Conference (ISOCC), Jeju, Korea, 3-6 November 2014; pp. 30-131.

7. Kolarik, V.; Lubaszewski, M.; Courtois, B. Towards self-checking mixed-signal integrated circuits. In Proceedings of the ESSCIRC'93: Nineteenth European Solid-State Circuits Conference, Sevilla, Spain, 22-24 September 1993; pp. $202-205$.

8. Axelos, N.; Watson, J.; Taylor, D.; Platts, A. Built-in-self-test of analogue circuits using optimised fault sets and transient response testing. In Proceedings of the Eighth IEEE International On-Line Testing Workshop (IOLTW 2002), Isle of Bendor, France, 8-10 July 2002; pp. 135-139.

9. Yuan, J.; Tachibana, M. A common-mode BIST technique for fully-differential sample-and-hold circuits. IEICE Electron. Express 2012, 9, 1128-1134. [CrossRef]

10. Fayomi, C.J.B.; Roberts, G.W.; Sawan, M. Low-voltage CMOS analog bootstrapped switch for sample-and-hold circuit: Design and chip characterization. In Proceedings of the 2005 IEEE International Symposium on Circuits and Systems, Kobe, Japan, 23-26 May 2005; pp. 2200-2203.

11. Pouya, P.; Ghasemi, A.; Aminzadeh, H. A low-voltage high-speed high-linearity MOSFET-only analog bootstrapped switch for sample-and-hold circuits. In Proceedings of the 2015 2nd International Conference on Knowledge-based engineering and Innovation (KBEI), Tehran, Iran, 5-6 November 2015; pp. 418-421.

12. Ballo, A.; Grasso, A.D.; Palumbo, G. Charge pump improvement for energy harvesting applications by node pre-charging. IEEE Trans. Circuits Syst. II Express Briefs 2020, 67, 3312-3316. [CrossRef]

13. Ballo, A.; Palumbo, G.; Grasso, A.D. A Review of Power Management Integrated Circuits for Ultrasound-Based Energy Harvesting in Implantable Medical Devices. Appl. Sci. 2021, 11, 2487. [CrossRef]

14. Dong, S.; Liu, M.; Zhu, Z.; Yang, Y. A high linear CMOS body effect compensation bootstrapped switch. J. Circuits Syst. Comput. 2015, 24, 1550032. [CrossRef]

15. Ballo, A.; Grasso, A.D.; Palumbo, G. Current-mode body-biased switch to increase performance of linear charge pumps. Int. J. Circuit Theory Appl. 2020, 48, 1864-1872. [CrossRef]

16. Wang, L.; Yin, W.J.; Xu, J. Dual-channel bootstrapped switch for high-speed high-resolution sampling. Electron. Lett. 2006, 42, 1275-1276. [CrossRef]

17. Rabuske, T.; Fernandes, J. A sar adc with a moscap-dac. IEEE J. Solid-State Circuits 2016, 51, 1410-1422. [CrossRef] 